\title{
Gamifying Intelligent Daily Environments through Introducing Fictionality
}

\author{
Mizuki Sakamoto and Tatsuo Nakajima \\ Department of Computer Science and Engineering \\ Waseda University \\ \{mizuki,tatsuo\}@dcl.cs.waseda.ac.jp
}

\begin{abstract}
A virtual form is an abstraction that enhances existing products and services by adding a layer to virtualize them. From a technical point of view, the current informat ontechnologies already make it possible to develop a virtual form using varibus techniques that represent information such as augmented reality technologies or ubiquitous display technologies. The virtual form can return a immediate feedback to a user so that it becomes a good design tool to develop games deployed in public spaces.

We believe that incorporating fictional stories into-nrtual forms offers a new possibility for gamifying and enriching user experiences. However, only technological approaches cannot offer an ideal solution to develop attractive products and services if people do not feel values on them, and the design patterns for vimal form is not well defined, especially, when a fictionality is incorporated. The design patterns needs lake into account a semiotic aspect of a virtual form. One key factor, in particular, is hom strongly we believe in the reality of a fictional story within the virtual form from the semiotic aspect.

This paper proposes some design patterns to integrate fictional stories into the real world for gamifying intelligent daily environments The proposed design patterns cover three aspects. The first aspect is to exploit visual reality. The second aspect is to exploit ideological messages in fictionat siories. The thind aspect is to compose multiple fictional stories. The paper also shows a case study to notivate and gamify to join the Haiku contest by increasing the awareness of the importance of the contest, and show the effectiveness of the proposed design patterns.
\end{abstract}

Keywords: Reality Semiotics; Fictionality; Design Activism; Role Playing; DigitalPhysical Hybrid Destgn, Gamification

\section{Introduction}

Our daly ife is becoming increasingly dematerialized and virtual as our surrounding daily artifac(s become more intelligent [16]. We define a virtual object as something that does not really exist but that has a real effect on our daily life as though it did. Baudrillard has explained our consumption behavior as consuming symbols that are associated with things, not the things themselves [3]. Because the symbolization of things will accelerate as we embed computers in our lives, our virtual consumption will progress rapidly.

In our daily life, fictional stories play an important role in offering rich user experiences. Theme parks, which consist of entertainment attractions, rides, and other events in a single location, exist for the enjoyment of large numbers of people. More elaborate than a simple city park or playground, a theme park usually provides attractions associated with popular fictional stories and is a typical example of how to integrate fictional stories into real-world 
activities. Disneyland is a typical, and the most famous, theme park. Many Disney characters appear at Disneyland. Each attraction at the park is immersively constructed based on a Disney story. Because those stories are very popular, when we are at Disneyland we feel that its characters exist in the real world, that we can meet those characters and that we can enjoy being with them during our visit [13]. At carnivals, fictional stories are also key to enhancing user experiences. Fictional stories also could potentially enrich user experiences during travel, at museums, and in advertisements. Although fictional stories are good tools for enriching users' experiences with products and services, those users need to feel that the objects, characters and events that appear in the stories - but do not exist in the real world-are still realistic.

Seichi Junrei is a typical geek culture activity in Japan, particularly related to Japanese animation, manga (comic) and games, in which people visit a local region from animation, manga and games. "Seichi" means "Holy Land", "Junrei" means "Pilgrimage" Typícal animation fans in Japan arrive at a specific location in the local regions, and take pictures with the same screen/angle as it appears in the animation and upload them to their blogs. The most important aspect of Seichi Junrei is that something is brough from the fictional story to the real world. The fans create new stories using these pictures and the virtual characters from the fictional stories and share them within their community. The local region also plans to attract people who like the story to visit the region to enjoy some extra stories of the original stories as the local region's promotion. This phenomenon is a very interesting example of harmonizing the real world and the fictional world.

Recently, a new opera entitled The End opened in Japan. In the opera, one lead actress is a virtual, fictional singer named Hatsune Mikw who is very popular in Japan. The scenes and performances in the opera are completely generated by computers. This opera has expanded the traditional concept of opera by using Hatsune Miku, a figure that is already very popular among Japanese youth, who feel thatshe lives in the real world. Her existence is realistic to them. In fact, her concerts are very popular, in them, with the use of augmented reality technologies, she both sing and dances ${ }^{2}$.

Fictional stories already play an important role in product advertisements because they increase the appeal of the advertised product [15]. Incorporating fictionality into stories enriches oa experience because stories can more easily translate and abstract real-world preanings in a manner that is easily and attractively understood. For example, fictional stories can represent either nonexistent or future stories. Such fictional stories can flexib y offer us a broad range of information using non-existent artifacts such as magic and mysterious creatures. It is also easy to embed ideological messages in fictional stories, thus making it possible to teach about the importance of various social issues. Specifically, in Japan, animations, video games, and comics are very popular. Even adults are very familiar with their stories, because those stories represent the essence of our lives. There are also several infrastructures emerging that create new stories from already-existing stories about characters such as NicoNico Douga. Exporting these emergent contents is one of the biggest tasks performed by Cool Japan 4 , Japan's national branding strategy.

There are many serious social problems that we need to tackle. However, we need a new approach to increase the efficacy with which we can attack those problems. Incorporating

\footnotetext{
${ }^{1} \mathrm{http}: / /$ theend-official.com/

${ }^{2} \mathrm{http}: / / 5 \mathrm{pb} . j \mathrm{p} / \mathrm{mikupa} /$

${ }^{3} \mathrm{http}: / /$ www.nicovideo.jp/

${ }^{4}$ https://www.ana-cooljapan.com/
} 
fictionality into the real world makes it possible for a person who is struggling with a problem to play a role, because the story makes us believe in the importance of tackling social problems, making it possible to increase our positive thinking and become activists. Because a typical fictional story describes brave heroes, dignified heroines and mysterious magicians whose strong self-efficacy enables them to achieve difficult goals, when we play these roles in the real world, our attitudes and behavior are altered and our own self-efficacy increases. By expressing their messages through various artifacts that surround us, stories can be embodied into the real world. Our daily life is becoming increasingly complex, and we need to manage significant amounts of information every day. Thus, it is difficult to convey important ideological messages without presenting a large amount of additional information. For example, education is a traditional, heavily weighted method for teaching the importance of ideological messages that take a long time to learn. We need a new approach to alter our attitudes and behavior without the need to assess a large amount of information.

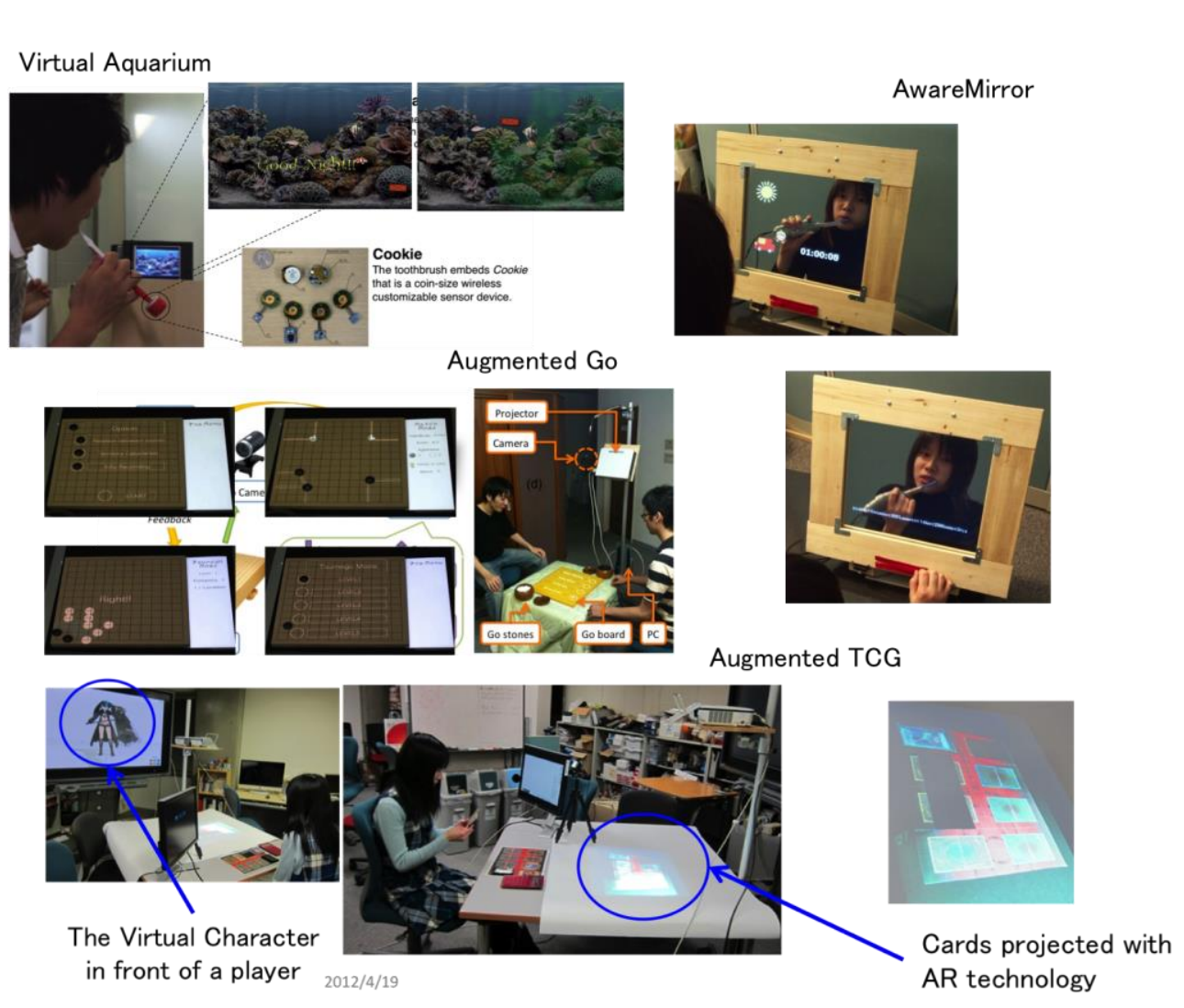

Figure 1. Virtual Forms

A Prtual form is an abstraction that enhances existing products and services by adding a layer to virtualize them. Virtual forms offer the potential to integrate fictionality into products and services to offer immersive experiences [22]. These virtual forms show dynamically generated visual expressions containing information that both provokes a person to feel that the artifacts have some additional value and enables him or her to consider the activities that use the artifacts as more attractive. For example, incorporating visual expressions into a display or projecting some information onto an artifact adds computational visual forms to existing daily artifacts. In past studies, several intelligent artifacts incorporating virtual forms, as shown in Figure 1, 
have been developed and their effectiveness has been assessed [9, 20, 25, 34]. For example, AwareMirror [9] provides an ambient presentation of personalized information on a mirror. Virtual Aquarium [20] presents a computer-generated aquarium in which the fishes' behavior is affected by a person's tooth-brushing behavior. Similarly, Augmented Trading Card Game [25] shows an empathetic virtual character whose behavior reflects an opponent's gaming behavior. These artifacts incorporate fictionality to increase a person's empathetic experiences. In [11], two properties-agency and immersion-are essential for developing more persuasive expressions ${ }^{5}$. Virtual forms offer the agency property through interactiveness with artifacts. In addition, sensing our environments and presenting feedback information in a pervasive manner enables virtual forms to react according to a person's current situation. This provides the property of immersion, because a person feels that expressions shown on virtual forms are a part of his or her world. The literature has shown that virtual forms can offer powerful persuasive effects, and not only because they increase empathy.

To embody fictionality in the real world, some insights into how pervasive games $[14,18]$ or alternate reality games $[17,32]$ blur the real and fictional worlds will be key to achieving better integration of those worlds. Originally, pervasive games were developed for fun, offering, for example, treasure hunts. However, a recent trend in pervasive games, such as World Without $\mathrm{Oil}^{6}$, allows users to try to solve serious, realworld social problems. Because designing activism-related games provides a new possibility to create a more desirable lifestyle by altering our attitudes and behaviors [10], pervasive games provide a promising approach to changing our world through activism. Alternative reality games are apromising approach for conveying messages to users using multiple channels. Fictional stories are embedded into a game that is played in the real world and that uses multiple channels. The channels offered in the game are used to exploit the game's fictronal story For example, in Perplex City ${ }^{7}$, trading cards are used to introduce the characters and story. Web sites, emails, phone calls, and SMS messages are then cooperatively used tosolve riddles in the mystery story. Because the media is tightly integrated with our daily activities, we feel that the fictional story is realistic. For example, social Media such as Twitter and Facebook have become very popular. Thus, fictional stories that are embedded in social media increase the feeling that the stories are occurkingin the real world.

One of the most important factors in embodying fictionality in the real world is roleplaying, in which a person plays a fictional role in the real world without losing his or her grasp on reality. This type of role-playing is called pervasive role-playing [19]. A person's fictional experience becomes tangible if he or she feels that the embodied fictionality is realistic. Reality is the most important criterion for success in pervasive role-playing. As described In [18], the most important factor in pervasive game design is to break the magic circle (in terms of space, time, and the social world) when people play fictional roles different from their roles in daily life. The boundary that the magic circle creates between fact and fiction should be taken into account when embodying fictionality in the real world.

Because virtual forms present a part of a fictional story by showing expressions, a person who pervasively plays a role to achieve his or her goal can easily remember the story [25]. In addition, as a part of the story, virtual forms can return appropriate feedback to alter a

\footnotetext{
${ }^{5}$ His discussion mainly focused on videogames, because videogames offer very strong persuasiveness.

${ }^{6} \mathrm{http}: / /$ worldwithoutoil.org/

${ }^{7} \mathrm{http}: / /$ perplexcitywiki.com/wiki/Main_Page
} 
person's attitude and behavior at any places. This means that virtual forms are desirable tools to incorporate fictional stories into the real world through the use of pervasive games, and a person can play a role in the story through expressing its properties of agency and immersion clearly [26, 27]. However, we need design patterns, similar to those that have been proposed in the architecture field by Alexander [2], to develop persuasive virtual forms in a systematic fashion. In this paper, we do not discuss design patterns as a way to cause a person to lose his or her belief in reality by seamlessly blending fictional and real worlds to make the magic circle vague in terms of space, time and the social world. Rather, our approach is to extract these design patterns from three cases. The first case extracts design patterns to increase a sense of visual reality of fictional elements. We chose the second case because we believe that using ideological messages in fictional stories is essential to increase the persuasiveness of virtual forms. The third case was necessary because although different persons prefer different stories, these stories must be combined into one when multiple persons participate to achieve the same goal. In our approach, we are not concerned with the ontology of fictionality presented on virtual forms, but we rather focus on semiotic aspects of fictionality [7, 37]. This means that we discuss the meaning of fictionality so it does nol matter whether incorporated fictionality really exists or not.

The contributions of the paper are as following.

- Ubiquitous computing researches have proposed many techniques integrate virtual worlds and real worlds, but good design patterns have not been proposed from a media design aspect. The proposed design patterns supplement traditional researches to design better ubiquitous computing environments.

- Using fictional stories is a promising direction to navigate people towards better lifestyle $[26,27]$. A sense of reality of fictronal stories is essential to realize better navigation of human attitude and behavior As described in [24], human navigation is essential to solve various serious social problems that we are currently facing with.

- We mainly use Japanese anlmated movies that contain various advanced visual techniques to increase a sense of reality of fictional elements. The paper shows that the power of Japanese animated movies cam be an interesting research topic for exploiting future ubiquitous computing researches.

In the remainder of this paper, we first describe semiotic objects. We extract the design patterns from three case tudies, as described in Section 3. We also explain why the design patterns are important for incorporating functionalities into the real world. In Section 4, we show how design patterns can be used to design virtual forms. This section also presents those elements that are necessary to maintain reality. In Section 5, we present several issues for future research, and conclude the paper.

\section{Semiotic Objects}

The result of semiosis allows us to deal with non-existing objects. In our mind, we can create various non-existing objects as semiotic objects. The semiotic object is

[...] every device by which an expression conveys a set of properties as its content $[\ldots]$ all expressions which convey as their proper content whatever we are used to call the meaning of the signified of the expression: the idea of an animal, of a place, of a thing, of a feeling, of an action, of a natural law like universal gravitation, of a mathematical entity, et cetera. (pp. 88-89 [7]) 
Symbols are used to represent non-existing objects to express fictional objects, abstract objects, or objects in alternate histories. Since these symbols are used to represent a variety of things, creatures, and worlds to express non-existing objects, the semiotic approach is suitable as the basis of the analysis of the reality on fictional stories.

Our analysis and extracting design patterns are based on the above conceptualization because most of fictional stories contain non-existing objects, and we feel a sense of the reality on the non-existing objects when their meanings are clear and understandable and there are plausible reasons that these objects exists in the stories.

\section{Extracting Design Patterns}

We convened three focus groups to extract useful design patterns for designing virtual forms that incorporate fictional stories. Each focus group involved 20 participants and a discussion that lasted for a few hours. The participants were university students whowere 2124 years of age. All of them were Japanese and were very familiar with recent Japanese animations, games and product promotions. After watching the movies or playing the games described in the following sections, the participants discussed whether the movies and games gave them a sense of reality and which major elements brovided that sense of reality. Two media analysis research experts chose the movies and games accoiding to the main theme of each focus group. Finally, the experts extracted design patterns from the discussions in the focus groups. In Section 3.1, we discuss the design patterns that allow a user to feel the reality of fictional stories when the stories are used for product and service promotions. It is essential that a user feels that the products exist in the real world and they are very attractive. In Section 3.2, we present design patterns for using ideological messages embodied in a fictional story to increase its persuasiveness related to behavior and attitude changes in the real world. In Section 3.3, design patterns foldomposing multiple fictional stories consistently are presented with the goal of supporting each person in playing his or her favorite role, or possibly different roles.

\subsection{Design Patterns to Exploit Visual Reality}

Animated movies are very useful tools because they make it easy to offer fictional worlds and characters. With animation, we can offer empathetic fictional creatures and marvelous futuristic worlds that are attractive to the user. It is easy to embed ideological messages that represent human dreams and expectations into animated stories. In addition, the typical Japanese animated stories are full of positive thinking, so the stories can be enjoyable while increasing our self-effreacy and helping us to overcome difficult problems.

Currently, typical product promotions use empathetic characters that appear in popular animated stories as tools to increase the attractiveness. For example, Pokémon ${ }^{8}$ characters are widely used to promote foods for kids, such as a retort-packed curry food and bread. This animated movie is very popular with most Japanese children, so a promotion broadcast within the animated television movie is effective way to make these products appealing to these children. Although the promotion may increase the buying impulse of children, it does not convince them that the products are attractive. Thus, the products will be forgotten when they become adults.

In this subsection, we present four design patterns for promoting commercial products by using fictional stories. The design patterns were extracted from discussions in the first focus group, while watching several animated movies for product promotions.

${ }^{8} \mathrm{http} / / /$ www.pokemon.com/us/ 


\section{I-I. Offering Vivid Visual Impact or Surprise Attracts us to the Products.}

Pepsi NEX uses Cyborg 009 for their product promotion ${ }^{9}$, and Tiger \& Bunny ${ }^{10}$ uses several Japanese company logos, such as Softbank ${ }^{11}$ and Bandai ${ }^{12}$, to promote their company brands. In the Pepsi NEX promotion, the cyborg heroes move very quickly, which becomes a metaphor for the Pepsi NEX's sharp taste. Additionally, a pretty heroine creates an empathetic feeling for the products. In the animated story of Tiger \& Bunny, justice and heroics are commercialized commodities. Some people choose to become costumed superheroes, and each is sponsored by an actual major company, which is featured as an advertisement on the heroes' uniforms. These superheroes are perceived as "cool" and become metaphors implying that the companies are also "cool". These vivid expressions of an unusual daily life surprise people, which generates strong and memorable impressions of the target products.

\section{I-II. Offering a non-fiction Story that Makes us Believe the Promotion.}

TAISEI Corporation ${ }^{13}$ promotes its brand image using an animated movje ${ }^{14}$. In the movie, a woman is working on the construction of the Bosporus tunner. The non-fiction story explains that her work contributes to an amazing construction that will appean in the world map. The movie demonstrates that the company has achieved this amazing work, so the audience for the movie can own the positive feelings about the company through the amazingly realistic scenes of the tunnel. The promotion is very usefal because most of us do not know that the TAISEI Corporation has built these amazing constructions âl over the world.

\section{I-III. Offering a reality that makes us believe the fict onal story in the promotion.}

DOCOMO's ${ }^{15}$ promotion, Xi AVANTT, shows a vision of the future mobile phone; this promotional movie uses several reahistic landscape scenes of present-day Barcelona. The reality of the background landscape scene offers a feeling that the vision told in the fictional story will be realized in the near future real world.

The Tokyo Disney Resort's promotion 17 reminds each person of a real memory from when she previously visited the Tokyo Disney Resort. So the story shows that she will enjoy visiting Tokyo Disney Resort even when she becomes old. Therefore, we believe that the happy memories from the Tokyo Disney Resort will be inherited by our children.

\section{I-IV. Offering empathy that attracts us to the products in the promotion.}

It is typical to use animated characters to create empathy in promotion videos. As described previously, Pokémon characters are used to promote various commercial products for kids. Additionally, the Japan Racing Association uses characters and giant humanoids in Evangelion, which is a very popular animated movie that is liked by many young Japanese adults $^{18}$. The purpose of the promotion is to promote horse racing to young adults.

\footnotetext{
${ }^{9}$ htto://009.ph9.jp/pepsi-nex/

${ }^{10} \mathrm{http} . / /$ tigerbunny.wikia.com/wiki/Tiger_\%26_Bunny_Wiki

${ }^{11} \mathrm{http}: / / \mathrm{mb}$. softbank.jp/en/

$12 \mathrm{http}: / / w w w . b a n d a i . c o . j p / \mathrm{e} /$

${ }^{13} \mathrm{http}: / /$ www.taisei.co.jp/english/

${ }^{14} \mathrm{https}: / / \mathrm{www}$.youtube.com/watch?v=OKoCl-3E0Vw

${ }^{15}$ DOCOMO is one of the biggest mobile telecom companies in Japan.

${ }^{16} \mathrm{https}: / / \mathrm{www}$.youtube.com/watch?v=IP5nAkG51ME

${ }^{17} \mathrm{https}: / / \mathrm{www}$.youtube.com/watch? $\mathrm{v}=\mathrm{clFq} 7 \mathrm{xwxV}-\mathrm{Q}$

$18 \mathrm{https} / / / \mathrm{www}$. youtube.com/watch? $\mathrm{v}=$ toEcz4inet8
} 
Animated stories are preferred across generations in Japan, but each animation covers only a specific target generation. Thus, an animation that is of interest to a specific generation can be used to appeal to a different generation who may not have a strong interest in the target products. The first focus group also analyzed four promotional movies that use the original animated stories. The movies are both successful and unsuccessful at promoting products and company brands. We consider how the movies fit the design patterns explained in this subsection.

The first is the promotion movie for Meiji fruits gummi candy. The story is named Megumi and Taiyo Tweet Love Story ${ }^{19}$. In the story, a heroine Megumi eats a grape gummi candy when something needs to be considered deeply. The scene fits to design pattern I-I. When using a fictional story, it is not easy how the audiences feel the reality on the story in accordance with design pattern I-II. Tweet Love Story uses a social media, Twitter, to make us feel the reality of the story. The audience can talk with the story's hero Taiyo via Twitter. Taipro gives us answers when we gave him some advices on how to get closer to Megumi. Megumi and Taiyo also talk with each other on Twitter so everyone can know their conversation. The audiences' advices have strong impacts on the conversation between Megumi and Tdyo: This means that the story changes its ending according to our advices to Tavyo, and the fact feels us that the story is realistic in our world.

Taiyo is working at a vineyard, and there are some scenes showing that grapes are healthy and delicious. This gives enough information showing the product's excellence in accordance with design pattern I-III. Finally, the story chooses a character designer whose characters are recently very popular in many media. Thus the audience easily feels the empathy on the characters even though the story and its characters are ofiginal. Then, design pattern I-IV is satisfied.

The second is a promotional movie named Mercedes-Benz Next A-Class ${ }^{20}$, which promotes Mercedes-Benz's new A-Class cars. In the story the promoted car is depicted in a near-future world. The speed of the car is nidely shown in the movie, fitting with design pattern I-I. Additionally, the movie shoys that the ear offers very high performance, which fits design pattern I-II. In the animated movie, the presentation of the car is very realistic. Additionally, the story is about finding legendary ramen noodle shop. ${ }^{21}$ Finding a nice ramen noodle shop is a very popular activity for young Japanese adults. Thus, the story is particularly realistic for the young adyits who are the target users for the A-Class cars, which fits design pattern I-III. Finally, the movie adopts a claracter from Evangelion, and most young males know and like Evangelion. Evangelion is one of the most popular animations in Japan, and its characters are well known. The people who like Evangelion's characters also like the characters in Next AClass, which fits design pattern I-IV.

The third promotional movie is for Subaru, which is a Japanese automobile company. This promotional movie is named Wish Upon the Pleiades ${ }^{22}$. This promotion is very interesting because there are very few connections to Subaru in the movie. The name of the main heroine is Subrort but the movie does not show any cars in the story. However, the characters in the story and the story itself are vividly attractive to many Japanese traditional animation fans. The story is based on a magic girls' story, and many scenes in the movie are very typical of a magic girls' animated movie. Thus, the movie creates empathy with many animation fans, and the characters in the movie have become very famous in the avid animation communities.

\footnotetext{
${ }^{19} \mathrm{http}: / /$ www.meiji.co.jp/sweets/candy_gum/fruits_gummi/part1/

${ }^{20} \mathrm{http}: / /$ next-a-class.com/

${ }^{21}$ Ramen is a Japanese noodle dish, which is of Chinese origin.

$22 \mathrm{http} / / / \mathrm{sbr}-\mathrm{gx} . \mathrm{jp} /$
} 
The movie makes the name Subaru famous, although the company name may not be popular among young adults. The company has also opened several public festival events using the characters. Many young adults who are interested in the characters visit the events and learn more about the company. The original story follows only design pattern I-I and I$I V$, but design pattern I-II and I-III, which are not satisfied in the story, are compensated by the reality offered in real world attractions in festival events. This strategy shows a possibility for using transmedia storytelling for effective promotions in the future.

The fourth movie is Toyota's Peace Eco Smile ${ }^{23}$, which promotes its brand using an original animated story. In the movie, a young male person who comes from outer space tries to learn about products and rules in our world. In particular, the story uses a love story to explain that the technologies developed by Toyota are very eco-friendly. However, the characters in the story are not empathetic enough because the characters are very new to most people and the story is not long enough to allow viewers to develop empathy for the characters. The story does not give us enough information to understand that Toyota's technologies are superior. In addition, the background scenes are not realist ic enough, although there are a few impressive visual representations that provide us with metaphors showing Toyota's excellence. Thus, the promotion does not fit the proposed design patterns and has not been successfully accepted by most audiences

The above design patterns indicate that a sense of reality of real products is increased even when they appear in fictional scenes. The design patterns are essential to make fictional scenes more realistic.

\subsection{Design Patterns to Exploit Ideologicar Messages inf Fictional Stories}

Virtual characters are widely used in our daily life. For example, famous Disney characters such as Mickey Mouse and Dorald Duck proyoke empathetic feelings easily, anytime and anywhere. In addition, Hello Kitty and Pokémon are now found all over the world [1]. In animations and games, each virtual character has its own personality and story, which can be used as a medium to convey special information and messages. If people are familiar with the fictional story behind an animation or a game, then the story's characters will easily recall the story's leitmotif without,requiring much additional information beyond action or interaction among the characters.

In contemporary Japanese Society, several posters for public service announcements have incorporated virtal characters from recent popular animation stories. For example, $K$-ON! has been used for promoting a national survey in Japan ${ }^{24}$. In the story of $K$-ON!, high school girls try to realize their dreams with cooperative efforts among them. This becomes a persuasive message conveying the idea that everyone's participation is important for the national survey. Also, NFGD, which promotes guide-dog training, has created two posters using popular characters from Puella Magi Madoka Magica ${ }^{25}$. The girl in one of the posters is rebellious, but very considerate of her friends. The girl used in the other poster is very close to her friend, and thinks and cares about her friend even when they are far apart. Many young gils admire these two characters. Thus, the posters contain the implicit, strong, and persuasive message that a person can imitate these magic girls by becoming a puppy walker. This example shows the effectiveness of using virtual characters with established background stories to attract people. Moreover, the example provides good evidence that virtual characters can be used to convey ideological messages in a story that might play a significant

\footnotetext{
${ }^{23} \mathrm{http}: / /$ www.toytoyota.com/pes/

${ }^{24} \mathrm{http}: / /$ www.youtube.com/watch?v=IdAkKZKEfGU

${ }^{25} \mathrm{http}: / /$ www.nkoku.jp/pos
} 
role in changing attitudes. In Japan, most young people have enjoyed animation and game stories for a long time, and they are very familiar with popular animation and game characters. This awareness is, we believe, a good prerequisite for using virtual characters to enhance emotional feelings and successfully convey ideological messages with the goal of persuading people to change their behavior and attitude.

The second focus group discussed how two stories in well-known animation movies that were not originally intended to promote products could be used for the purpose, and debated how they could be used to do so. The extracted design patterns show how stories' ideological messages can be used to alter the behavior and attitude of a person related to his or her willingness to buy a product. Many Japanese animation stories use ideological messages about issues such as environmental protection or world peace to increase dramatic tension. ${ }^{26}$ Additionally, because stories provide numerous representations of the positivity in our future, they increase our self-efficacy with respect to accepting and understanding theif messages. This aspect of the use of stories is very important because it usually takes a verylong time for us to understand the importance of a message and to arrive at the belief that a story s message must come true. If we know these stories well already, the scenes or characters in the movies become metaphors for the story's messages and remind us of their importance without having to take the time to teach, as described in the previous paragraph.

If an actual, serious social problem such as sustainability can play a primary role in a fictional story, then design patterns are useful for encouraging people to critically think about the problem in the real world. The self-efficacy of a person why plays a role in the story to overcome the problem in the real world, and to have a strong positive feeling about embarking on a mission to attack that problem, also groys. Recent findings in the field of positive psychology have offered scientinc eviderce that positivity increases success in life [8].

The first movie was Celebi: A Timeless Encounter ${ }^{27}$. The ideological message in the story was that we should not selfishly violate forests and lakes. Celebi is one of the Pokémon ${ }^{28}$ characters; he is a god whoprotects a forest. A selfish person caught Celebi and used the character's power to destroy a forest and lake. Finally, Satoshi, who is the trainer of Pikachu (one species of Pokemion, reminded Celebi that he (i.e., Celebi) was their friend and that they had had joyful times together with the blessing of the forest and lake. In the end, the forest and lake were finally recoyered. The story showed that protecting forests and lakes is essential to maintaining our ecological system.

After watching the movie, the second focus group discussed what types of products could be promoted using characters or scenes from the movie. Some of the participants believed that cleaning products would be appropriate for such a promotion, because the recovery of a forest and lake could be a metaphor for making something clean. The focus group also discussed whether the movie could be used to promote eco-friendly products such as lowpower televisions. However, most of the focus group participants said that it would be difficult to find a metaphor in the movie that could promote eco-friendly products.

From the discussions, we gained the insight that one key issue is whether a movie provides a good metaphor for a target product. We usually remember the characters in a movie easily,

\footnotetext{
${ }^{26}$ For example, Nausica $\ddot{a}$ of the Valley of the Wind illustrates the dilemma between a natural way of life and military destruction of culture, land and resources. Similarly, the main theme of Earth Maiden Arjuna is the importance of organic farming to maintain the earth's sustainability, and that story's secondary theme relates to the disconnect between emotions, thoughts, words, and actions.

${ }^{27}$ http://en.wikipedia.org/wiki/Pok\%C3\%A9mon_4Ever

${ }^{28}$ Pokémon is a creature that appeared in a movie that was originally based on a computer game. The trainer of Pokémon trains his/her own Pokémon to fight against other trainers' Pokémons.
} 
as discussed in the focus group. In animated films in particular, the main characters usually have very clear characteristics with strong appeal, and thus, they become good metaphors.

The second movie was Re: Cyborg $009^{29}$. The ideological message of this story was that a miracle that solves hard social problems would occur if we wish hard, every day, for peace. Because evil lives in our minds, each person has the ability to start a chain of destruction. However, if we wish to stop that chain, a miracle will change our world and solve difficult social problems.

The second focus group discussed whether fair-trade products are appropriate for promotion using the scenes of Re: Cyborg 009, because justice is a key ideological message in the movie. Fair trade emphasizes social justice over profits and is about better prices, decent working conditions, local sustainability, and fair trade terms for farmers and workers in the developing world. Fair trade may close the large economic gap between developed añd undeveloped countries. Of course, because fair trade was not well-known by all of the participants in the focus group, we needed to explain it during our discussions. In addition, the scenes in the movie were not direct metaphors for fair-trade products. The participants in the focus group needed to think deeply about how fair trade was related to the movie's ideological message.

From our analysis of the second focus group's discussions, we extracted the following design patterns for incorporating ideological messages so that people will believe that those messages are also essential in the real world.

\section{II-I. Express The Story's Ideological Message Clearly.}

The most important issue is that pepple need to easily understand the story's ideological messages without need for additional explanation presented in other media such as books. In addition, a vivid or exaggerated presentation of the ideological message is essential to ensure that people retain a deep memory of that message

\section{II-II. Make the Participant Play a role in the Story.}

A user should be mvilved in the story to increase his or her sense of the story's reality. In the above examples, because a paticipant merely watches a movie, it is hard for him or her to have a strong feeling that story is a part of his or her real world. Therefore, interaction with the story should be explicitly ingorporated into virtual forms.

\section{II-III. The person's behavior should be consistent with the story's ideological goal.}

When a person plays role in a story, he or she behaves according to the characteristics of that role. His or her behavior should be consistent with the goal of the story's ideological messages. In the above examples, target products did not directly appear in the stories. Therefore, a person needs to create his or her own story in which he or she uses the target products; the actions that take place in his or her story must be consistent with the goals of the fictional stories.

\section{II-I Offer a metaphor to remind the viewer of the story's ideological message.}

A good cue is necessary to remind a person of the story. As described at the beginning of this section, in many advertisements, a character from the story becomes a cue to remind people of the story, and that character also becomes a metaphor to remind people of the story's ideological message. In addition, empathetic products may be used as a metaphor. In

${ }^{29}$ http://009.re-cyb.org/ 
other words, the story should offer impressive characters or products that become metaphors to remind people of its ideological message.

The ideological message typically contains some educational aspects. When applying design pattern II-I, it is essential not to lose the entertainment even if the ideological messages are clearly incorporated. Design pattern II-II indicates that the agency and immersion properties offered by virtual forms are essential to maintain a feeling of reality. Additionally, design pattern II-III is important because inconsistency between a person's behavior and the story's goal may cause a misunderstanding of the message. Similarly, when using a character as a metaphor design pattern II-IV requires care. If the character's role is not consistent with the story's ideological message, it may cause a misunderstanding of that message.

\subsection{Design Patterns to Compose Multiple Fictional Stories}

In the near future, it will be essential to mix multiple stories when integrating a fictional story into the real world; it is unlikely that the use of just one story will attract most people, because everybody has a different personality [23]. In particular, each person has his or her own favorite story and likes to play a role in that story according to his on her personality and past experiences. However, even though each person might have a different personality, everybody still must think deeply about serious social problems in the real world. Therefore, it will be important to mix together multiple stories that every user enjoys.

There are currently many cases of mixing cmultiple, existing stories. Avengers ${ }^{30}$, an American superhero film that came out in 2012, is an example of on story that consists of multiple stories mixed together. In Japan, some movies for children contain stories that their parents will like, so that the parents will 6oth enjoy the novie and be encouraged to take their children to see it. ${ }^{31}$

Once a video game player begins to love the particular content in a game or film, his or her feelings about other activities in real life in various situations are affected. For example, if a player likes a video game, he or she may eel a sense of closeness to a service that includes some content related to tha game. However, to attract people to a particular type of media, it is not enough to simply ntroduce a character from a story that originated with a different type of media. For example, a video game named E.T. The Extra-Terrestrial ${ }^{32}$, which was inspired by the popular Anferican science-fiction film E.T., is one example of such a failure. The goal of the game s to find parts of a communication device to contact E.T.'s companions in the mother star, and then to put them together, thereby enabling E.T. to return to the mother star to find the parts. In this game, E.T. falls into many pits. One player of the E.T. game said "I've seen the movie, and I don't remember ET falling into one pit, much less 100!" 33 . This comment shows that there is no consistency between the original movie and the subsequent game. Furthermore, this lack of consistency significantly decreases the feeling of the game's reality. When a story is reused in a new form of media, the story in the new media must be consistent with the original story so that the reality of the original media is maintained.

In the third focus group, we discussed design patterns that mix existing stories by analyzing a popular game named Super Smash Brothers ${ }^{34}$, which includes characters and scenes from other popular games. The Super Smash Brothers series, published by Nintendo, is a battle-based action game in which four players can join the game simultaneously. Many

\footnotetext{
${ }^{30} \mathrm{http}: / /$ marvel.com/avengers_movie/

${ }^{31}$ ex. Kamen rider ooo movies wonderful shogun and 21 core medals, http://www.tv-asahi.co.jp/ooo-gokai/

${ }^{32} \mathrm{http}: / /$ en.wikipedia.org/wiki/E.T._the_Extra-Terrestrial_\%28video_game\%29

${ }^{33} \mathrm{http}: / /$ videogamecritic.net/2600de.htm

${ }^{34} \mathrm{http}: / /$ www.smashbros.com/en_us/index.html
} 
famous Nintendo characters, such as Mario ${ }^{35}$ from Super Mario Brothers and Pikachu from Pokémon, appear in the game, and a player can choose his or her favorite character and control it to participate in the battle. Although each character has a different background story, it is natural that they battle with each other on the same stage in the Super Smash Brothers world. The game offers players new, exciting experiences in the Super Smash Brothers world. Additionally, the game provides players with an increased opportunity to learn about other game titles. In fact, a significantly large number of players have developed an interest in the other game titles mixed into Super Smash Brothers, with which they were previously unfamiliar.

The analysis of the game that emerged from the discussions in the third focus group led us to extract the following design patterns for realizing a natural cross-story game.

\section{III-I. Retain Characters' Traits from the Original Stories.}

In Super Smash Brothers, the existing characteristics of each character are extremely clear and it is easy for players to perceive them. These characteristics are not vistal, the motion and effect of each character's waza ${ }^{36}$ should be the same as in the original games. If each character's presence becomes unfamiliar because muliple characters have been mixed together, the game loses its attractiveness to existing fans as a result of having mixed multiple stories.

\section{III-II. Use Background Scenes from the Original Stories.}

Any worlds used in a game that mixês multiple stories should be the same as in each original story. Some games use battle stages, including ginmicks or items, which can easily remind players of the original games. For example, when a person who has played Pokémon plays Super Smash Brothers, the \$tage related to Pokemon reminds him or her of some scenes from the Pokémon story. This familiarity inçreases the player's nostalgia, which leads him or her to feel the value of playing a game that mcludes the Pokémon story. The background scene of each stage can workas a metaphor to strongly engage the player.

\section{III-III. Keep the Characters' Goals from their Original Stories.}

In a game, each character has his or her own goal that must also achieved by its player. When multiple stories originating from existing games are mixed together, the goal of a character in the new, mixed story should be consistent with his or her goal in the story of the original game. The main theme of Super Smash Brothers is a battle among well-known, attractive characters. Thus, all games mixed into the Super Smash Brothers story contain, to a greater or lesser degree, battle factors. For example, Mario battles against an opponent during his adventure and Pikachu uses thunder power to help a partner against his opponent. Winning the battle is one of the important goals in these games. Thus, it is easy to maintain the consistency of a character's goal in both Super Smash Brothers and the characters' original stories. The consistency of each character's goal in both a mixed story and an original story $\mathrm{p}$ lays an important role in maintaining the reality of the new game's mixed story.

\section{III-IV. Have a Reason to gather the Characters from the Original Stories.}

There is an original story behind Super Smash Brothers. The story is that "each character's figure in a toy box starts to move by magic." Usually, when many characters from different backgrounds appear in the same world together, it does not feel natural to enthusiastic players

\footnotetext{
$35 \mathrm{http}: / /$ mario.nintendo.com/

${ }^{36}$ Waza are skills and moves unique to a certain fighter or fighting style.
} 
of the existing games. In particular, it is a little bit strange when characters appear in the world of a different game. However, in the real world, it is not strange for many characters' figures to coexist in a toy box.

The above design patterns can work as a tool to mix multiple stories and can help to reduce any sense of incongruity.

Every person has "my stories" that are the person's own stories, where he or she plays an important role. The design patterns can be used to compose "my stories" and original fictional stories not to violate a sense of reality of the original fictional stories. A new fictional role is defined for the person in "my stories", and the person plays the role in the stories. The stories are different from the original stories, but the basic atmosphere should be the same in these stories. Also, "my stories" are closely related to the real world. Thus, the person feels that he or she is involved in the fictional story if the design patterns described in the section are satisfied.

Our approach is also useful for analyzing a story that mixes multiple existing stories. For example, McDonald's sells Happy Meals ${ }^{37}$, which include toys featuring characters from various stories. Currently, there is no interaction among the characters from different stories. However, our approach offers a promising way to enhance the consumer experience by consistently mixing stories with multiple characters.

\section{Case Study: Gamifying Haiku Contest with Proposed Design Patterns}

The Life and Blood Donation Haiku Contest created public posters that featured Pokémon characters. The ideological messages of the Pokemon story are mutual help, friendship, a fatal encounter, and irreplaceable life. The posters included certain keywords to present these messages. Thus, contest applicants were reminded of the importance of those messages by their memories of enjoying the Pokemon story. In this section, we discuss how the design patterns described in the previous section can enhance event promotion by using virtual forms.

First, we consider whether to replace a static poster with an interactive poster incorporating virtual forms that dŷnamically express the Pokémon story. The interactive poster offers a game-like interaction with a person to increase the persuasiveness in a manner similar to that employed by advergames [31]. For example, people feel a sense of reality if movies offer some cues that satisfy design patterns.

We consider how to ure these design patterns presented in Section 3.1. Pursuant to design pattern I-I, some expressions in virtual forms should offer strong impressions to the person who plays a role in the story. For example, the person's Pokémon could grow and use special skills that show special effects. To satisfy design pattern I-II, a landscape that is familiar to the person could be presented. For example, we already have considered both singing Haiku and helping blood donation, and the landscape that presented these activities has a strong connection to the real world. Thus, design pattern I-III is satisfied. Most Pokémons are empathetic virtual characters, so the Pokémon example also satisfies design pattern I-IV.

Next, we consider the design patterns described in Section 3.2. Because Pokémon story contains clear ideological messages as described above, the design pattern II-I is already satisfied. One of the most important attractive features of virtual forms is enable us to interact with people. To satisfy design pattern II-II, the interactive poster offers a type of a game that explains both $\mathrm{Haiku}^{39}$ and the importance of blood donation. In the game, a Pokémon trainer

\footnotetext{
${ }^{37} \mathrm{http}: / /$ www.happymeal.com/en_US/index.html

${ }^{38} \mathrm{https}: / / w w w . k e n-h a i k u 2012 . j \mathrm{p} /$

${ }^{39} \mathrm{~A}$ haiku is a Japanese poem of seventeen syllables.
} 
who is also a Haiku poet teaches a person, who also interacts with the poster, to write a good Haiku. That person also plays the role of a Pokémon trainer, and they meet another Pokémon trainer who helps with blood-donation activities. Formulating their common goal can satisfy the design pattern II-III. The goal of the story is mutual help and friendship. The person helps the Pokémon trainers of a Haiku poet and a blood-donation volunteer to achieve their dreams. The Pokémon story is a typical hero's journey tale [5], and Pokémon trainers help each other to mature and to become self-actualized. By helping the other trainers to achieve mental growth, the person also becomes interested in both singing Haiku and blood donation. Finally, to satisfy design pattern II-IV, the trainers' Pokémons, or a typical landscape in the story, can be presented on the interactive poster as a metaphor to remind viewers of the message of Pokémon story by expressing it in virtual forms.

We need to consider the design patterns described in Section 3.3 when another person prefers a different story. Satisfying design patterns III-I and III-II is easy because characters' behavior or landscape scenes in one story can be easily maintained in the other story. For satisfying design patterns III-III, the ideological messages of multiple stories should be consistent. According to design pattern III-IV, it is necessary tô have one meta-story to evidence that multiple stories can coexist. In Japan, many people have gamified animation, comic and game cultures. As shown in [28], it is a type of a game to create a metastory for people who participate in social network cultures such as NicoNice Douga and Pixiv ${ }^{40}$. Therefore, one promising approach is to rely on geek and creative communities to create a metastory, because these communities have found that a consistent world in which multiple stories coexist results in the emergent evolution of content culture by creating a new story through the reinterpretation of existing stories. This phenomenon is typical in young Japanese people's animation and game culture, which supports advanced content culture.

We strongly believe that complete photorealistic and tangible immersion, such as that depicted in Star Trek's holodeck 4 , is the holy Conail of making embodied fictionality seem realistic. As described in Section 3.1, we have presented several analyses showing that complete photorealistic and tangible immersion is not always necessary for simulating reality when fictionality is embodied in the real world. However, there are some other examples to show that it is easy to lose touch with reality in a fictional story. Even the advanced technologies of the present do not allow us to embody fictionality into the real world with complete photorealistic and tangible immersion. Therefore, it is important to research how collecting andincreasing design patterns make people feel the reality of embodied fictionality.

We developed a simple paper prototype to realize the above example, and conducted a simple "Wizard of Oz" experiment based on the prototype using five participants who were Japanese university students. Some of the participants said: "It was an interesting approach to advertise a Haiku contest. The interactivity offered by the interactive poster gave me a deeper impression than the traditional poster"; "I like Pokémon, so it was great fun for me to play the interctive poster, and I could understand the importance of blood donation"; and "Virtuaforms that are pervasively embodied in our daily environments offer an immersive experience of feeling that the fictional story was more realistic. Additionally, the interactivity increased the sense of reality by playing a role in the story." In addition, one of the participants said, "I like to create a new story from existing stories. The story needs to define an ideal situation for me by considering a new interpretation of existing stories. The stories' metastory offered a consistent world that was comfortable for me, and it was fun for me to

\footnotetext{
${ }^{40}$ http://www.pixiv.net/

${ }^{41} \mathrm{http}: / /$ www.startrek.com/database_article/holodeck
} 
create the metastory on a social network.". In the next step, we develop a more complete working prototype and a larger user study to validate our approach.

\section{Conclusion and Future Directions}

The discussions described in this paper present several important design patterns to enhance the reality of fictionality embodied in the real world. There are two promising future directions to enhance our approach. The first direction is to use transmedia storytelling [6,21] to define the meaning of a visual object expressed in a virtual form by using information that has been expressed in other media. The second direction is to offer stronger persuasiveness to satisfy the design patterns described in the paper. A concept named procedural rhetoric $[4$, $12]$ is a promising way to discuss the issue in a more formal way.

Using fictional stories in transmedia storytelling is a promising approach for enhanding virtual forms. Transmedia storytelling allows a fictional story to be harmonized with the real world by fragmenting the story into multiple media presented at various locations in leveryday life. In this case, virtual forms are installed everywhere to embed, the fictional stories in the real word. The virtual form becomes a layer to enhance the real world through transmedia storytelling. The story embedded in the virtual forms virtualizes oun real world and offers additional enrichment. This approach offers the poential to enhance dally life experiences, and the stories that are immersively embedded in our world encourage us to solve various social problems anytime, anywhere. However to successfully integrate fictional stories, people will need to feel that those stories are realistic. Past studies show that achieving reality is an important design criterion for successfulintegration when incorporating virtuality and fictionality in the real world $[29,30]$. If a person does not feel the reality of the fictional stories, he or she will feel that any additional values offered by virtual forms do not exist in the real world [26, 27]. The direction is also useful to discuss how fictional stories can be translated for use in the context of different cultures [28].

Currently, one of the leading theories or how the computational visual form persuades people is Ian Bogost's concept of procédural rhetoric [4]. Bogost has explained that among persuasion strategies embodied in media such as movies and books, procedural rhetoric offers the most powerful persuasion effects. Virtual forms are an underlying infrastructure to offer procedural hetoric. Video games usually offer fictional worlds that are separate from the real world, but virtual forms are immersively embodied in the real world, and fictional experiences are lightly in eg ated into our daily, typical experiences. This means that virtual forms become effective tools to influence people's attitudes and behavior to alter their lifestyle in a way that is more desirable and meaningful. For designing effective virtual forms, it is important to discuss how the design patterns extracted in this paper should be integrated into procedural rhetoric for designing more persuasive and realistic virtual forms.

\section{References}

[1. Allison, "Millennial Monsters: Japanese Toys and the Global Imagination", University of California Press, (2006).

[2] C. Alexander, "A Pattern Language: Towns, Buildings", Construction, Oxford University Press, (1977).

[3] J. Baudrillard, "The Consumer Society: Myths and Structures", Sage Pub. Ltd., (1998).

[4] I. Bogost, "Pervasive Game: The expressive power of video games", MIT Press, (2007).

[5] J. Campbell, "The Hero with a Thousand Faces The Hero with a Thousand Faces", New World Library, (2008).

[6] C. Dena, "Transmedia Practice: Theorising the Practice of Expressing a Fictional World across Distinct Media and Environments", Dissertation Thesis, University Sydney, (2009).

[7] U. Eco, "On the Ontology of Fictional Characters: A Semiotic Approach", Sign Studies, vol. 37, no. 1-2, (2009), pp. 82-97. 
[8] B. L. Fredrikson, "Positivity: Top-Notch Research Reveals the 3 to 1 Ratio That Will Change Your Life", Three Rivers Press, (2009).

[9] K. Fujinami, F. Kawsar and T. Nakajima, "AwareMirror: A Personalized Display using a Mirror", Proceedings of the 3rd International Conference on Pervasive Computing, (2005), pp. 137-150.

[10] A. Fuad-Luka, "Design Activism - Beautiful Strangeness for a Sustainable World", Earthscan, (2009).

[11] F. Gonzalo, "Rethinking Agency and Immersion: Playing with Videogame Characters", http://www.siggraph.org/artdesign/gallery/S01/essays/0378.pdf, (2001).

[12] T. Harper, "Rules, Rhetoric, and Genre: Procedural Rhetoric in Persona 3", Games and Culture, vol. 6, no. 5, (2011), pp. 395-413.

[13] Imagineers, "Walt Disney Imagineering", Disney Book Group, (2010).

[14] C. Magerkurth, A. D. Cheok, R. L. Mandryk and T. Nilsen, "Pervasive Games: Bringing Computer Entertainment Back to the Real World", ACM Computer in Entertainment, vol. 3, no. 3, (2005).

[15] A. S. Mattila, "The Role of Narratives in the Advertising of Experiential Services", Journal of Service Research, vol. 3, (2001), pp. 35-45.

[16] S. Marzano and E. Aarts, "The New Everyday View on Ambient Intelligence", 010 Publisher, Rotterdam. (2003).

[17] J. McGonigal, "Reality Is Broken: Why Games Make Us Better and How They Can Change the World", Penguin Press, (2011).

[18] M. Montola, J. Stemros and A. Waern, "Pervasive Games - Theory and Design”, Morgan Kaufmann, (2009).

[19] M. Montola, "Tangible Pleasures of Pervasive Role-Playing", In Proceedings of International Conference on DiGRA 2007, (2007).

[20] T. Nakajima, and V. Lehdonvirta, "Designing Motivation in Persuasive Ambient Mirrors", Personal and Ubiquitous Computing, vol. 17, no. 1, (2013), pp. 107-126

[21] M. N. Ruppel, "Visualizing Transmedia Network: Links, Paths and Pecipneries", Dissertation Thesis, University of Maryland, (2012).

[22] M. Sakamoto, T. Nakajima and T. Alexandrova, "Digital-Physical Hybid Design: Harmonizing the Real World and the Virtual World", Proceedings of the 7th International Conference on the Design \& Semantics of Form \& Movement, (2012), pp. 211-222.

[23] M. Sakamoto, T. Alexandrova and T. Nakajima, "Analyzing the Effects of Virtualizing and Augmenting Trading Card Game based on the Player's Personality", Proceedings of The Sixth International Conference on Advances in Computer-Human Interactions, (2013), pp. 348-357.

[24] M. Sakamoto and T. Nakajima, "Micro-Crowdfûndng. Achieving a Sustainable Society through Economic and Social Incentives in Micro-Level Crowdfunding, Proceedings of International Conference on Mobile and Ubiquitous Multimedia no. 29, (2013)

[25] M. Sakamoto, T. Alexandrova and T. Nakajima, "Augmenting Remote Trading Card Play with Virtual Characters used in Animation and Game Stories - Towards Persuasive and Ambient Transmedia Storytelling", Proceedings of the 6th International Conference on Advances in Computer-Human Interactions, (2013), pp. 168-177.

[26] M. Sakamoto and T. Nakajima "Gamifying Social Media to Encourage Social Activities with DigitalPhysical Hybrid Role-Playing", Proceedings of the 6th International Conference on Social Computing and Social Media, (2014), pp. 581-591.

[27] M. Sakamoto, T. Nakaj1ma and S. Akioka, “A Methodology for Gamifying Smart Cities: Navigating Human Behavior and Attitude (Proceedings of the 2nd International Conference on Distributed, Ambient and Pervasive Interactions, (2014), pp. 593-604.

[28] M. Sakamoto and T. Nakajima, "The GamiMedia Model: Gamifying Content Culture”, 6th International Conference on Cross-Cultural Design, (2014), pp. 786-797.

[29] M. Sakamoto, A. Yoshii, T. Nakajima, K. Ikeuchi, T. Otsuka, K. Okada, F. Ishizawa and A. Kobayashi, "Human Interaction Issues in a Digital-Physical Hybrid World", Proceedings of the $2^{\text {nd }}$ International Conference on Cyber-Physical Systems, Networks, and Applications, (2014).

[30] M Sakamoto and T. Nakajima, "A Community-based Crowdsourcing Service for Achieving a Sustainable Society through Micro-Level Crowdsourcing", Proceedings of the International Conference on Internet, Politics,Policy 2014: Crowdsourcing for Politics and Policy, (2014).

[31] J. H. Smith and S. N. Just, "Playful Persuaision: The Rhetorical Potential of Advergames", Nordicom Review, vol. 30, no. 2, (2009), pp. 53-68.

[32] D. Szulborski, "This Is Not a Game: A Guide to Alternate Reality Gaming”, Lulu.Com, (2005).

[33] J. Valsiner, "Between Fiction and Reality: Transforming the Semiotic Object", Sign Studies, vol. 37, no. 1-2, (2009), pp. 99-113.

[34] T. Yamabe and T. Nakajima, "Playful Training with Augmented Reality Games: Case Studies towards Reality-Oriented System Design”, Multimedia Tools and Applications, vol. 62, no. 1, (2013), pp. 259-286. 

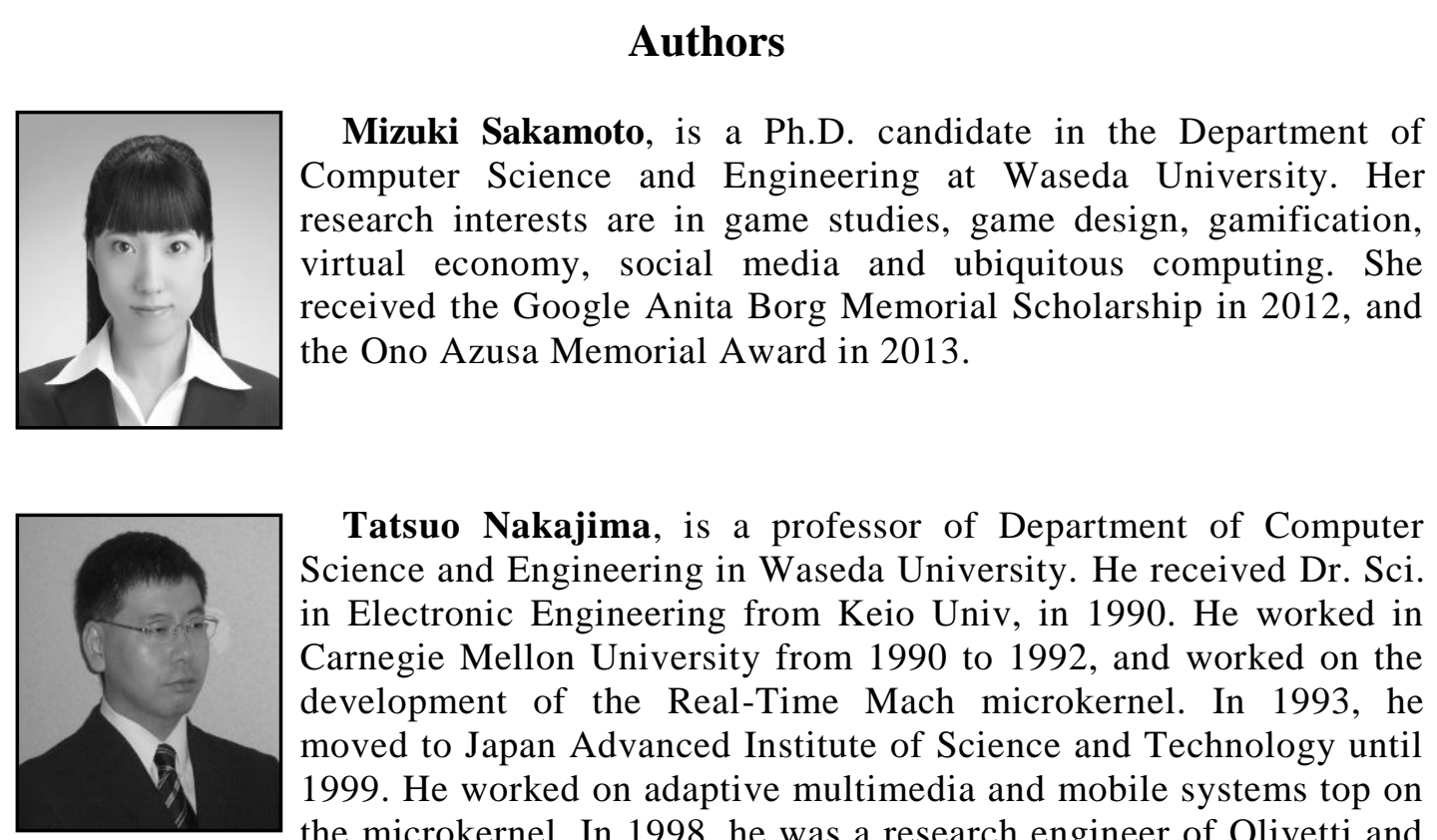

Tatsuo Nakajima, is a professor of Department of Computêr Science and Engineering in Waseda University. He received Dr Sci. in Electronic Engineering from Keio Univ, in 1990. He worked in Carnegie Mellon University from 1990 to 1992, and worked on the development of the Real-Time Mach miscrokernet. In 1993, he moved to Japan Advanced Institute of Science and Technology until 1999. He worked on adaptive nultimedia and mobile systems top on the microkernel. In 1998, he was a research engtneer of Olivetti and Oracle Research Lab., and worked on the implementation of the CORBA system. From 2000, he moved to Waseda University and worked on ubiquitous computing and operating systems for embedded systems His group has developed middleware for home appliances, AR-básed user interface, persuasive services and smart objects in the Ubicomp research. His group also developed a virtualization layer for multi-core processor based embedded systems. In 2005, he has a visiting research fellow of Nokia Research Center, Helsinki and a visiting professor of University of Helonki.

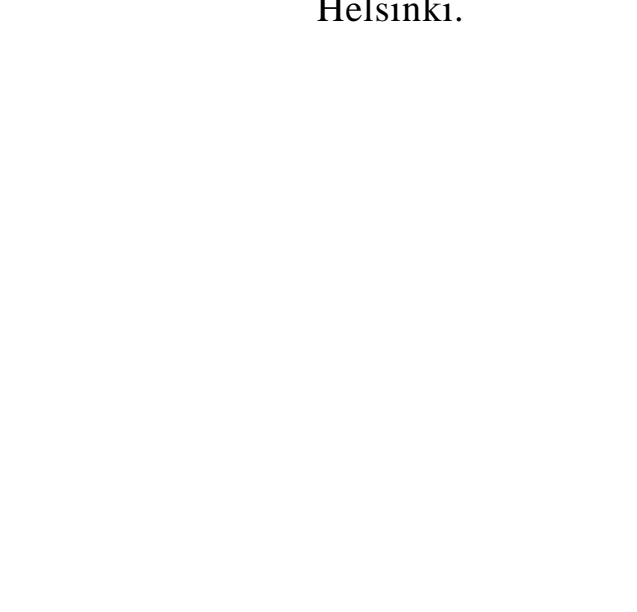

